\title{
Effectiveness of Virtualization in the Process of
}

\section{Running a Virtual Instance of a Computer System in a Layer Abstracted from the Actual Hardware}

\author{
Siddth Kumar Chhajer ${ }^{1}$, Rudra Bhanu Satpathy ${ }^{2}$ \\ ${ }^{1}$ MBA,Marketing, St. Peter's University, Chennai \\ 2 .M.Tech,Electrical, Electronics and Communications Engineering, St. Peter's University Chennai. \\ ${ }^{1}$ siddth2011@gmail.com, ${ }^{2}$ rudra@ gmail.com
}

\begin{abstract}
Under this research paper many different kinds of viewpoints concept of Virtualizations, the use of visualization for security reasons, and the Importance of IoT in the virtualization process. Moreover, this paper will signify that Different virtual environments are offered through a virtualized IT firm, which has to be accepted and operated if technology evolves quickly. This research paper will highlight many different types of strong and sustainable viewpoints about the visualization process and its impacts. It can be a supportive way for the next researcher to understand the scope of this research paper in a proper manner. In order to conduct a proper understanding of the effect of virtualization in running a virtual instance of a computer system from actual hardware will be the main aim of this paper. On the other hand, virtualization may also be described as a technique that can theoretically divide a server's physical resources and use it as several, separated computers known as virtual machines.
\end{abstract}

Keywords - Virtualization, Internet of Things, Technoarete Transactions, virtual networks, hardware and servers.

\section{Introduction}

In order to build a virtual system, virtualization employs software that replicates hardware capabilities. This enables IT firms to run many operating systems and numerous applications on a single server, and more than one virtual system. More efficiency and efficiencies also include the advantages of virtualization. Moreover, OS Virtualization is using software to enable a device to simultaneously run several OS images. The technique started decades ago, enabling managers to prevent costly processing power from being lost. Besides that, the quantity of the required hardware servers inside the organization and data center is reduced through virtualization as it also reduces the overall purchase cost and keeps huge quantities of hardware. In this research article, some important viewpoints will be evaluated in a proper manner such as Hypervisors detach, various virtual environments, and many other things.

\section{Literature Review}

\section{Concept of Virtualisation}

Virtualization utilizes software to create the abstraction of hardware on a computer, so that a computer's hardware parts, memory, storage, and more can separate into many virtual machines. As per the suggestions of Muchagata and Ferreira (2018), each VM operates a system of its own and acts as if it is running only a part of the actual computer hardware behind it. In this current era, business IT architecture virtualization is standard practice, and cloud computing is also driven by technology to enhance security systems in a proper manner. Through a virtualized IT company, different kinds of virtual environments are presented as they must need to be adopted and administered if technology changes rapidly. On the other hand, it is also possible to describe virtualization as a technology that has the capacity to separate the physical resources of a server conceptually and to utilize it as various, isolated computers called virtual machines. 


\section{Use of visualization for security reasons}

Practitioners of information security are overwhelmed with information. Network data, such as scan reports, viruses, worms, and spam blows are available. There are complaints from host and login systems users who have not updated their passwords, locked users, and users who are just suspicious as it supports deployment and patch management systems reports. As per the words of Chatzimparmpas et al. (2020), practitioners of information security are overwhelmed with information. Network data, such as scan reports, viruses, worms, and spam blows are available. On the other hand, there are complaints from host and login systems users who have not updated their credentials, banned users, and users who are just suspicious as it supports deployment and patching tools information is available.

\section{Advantages and disadvantages of virtualization}

\section{Advantage}

Since virtualization does not need the usage or installation of actual hardware components, it is a cheaper solution for IT infrastructures to deploy man ware-based anti-virus that directly enhances the security performance in a strong manner. Bernstein (2019) stated that large amounts of space and enormous monetary investments are no longer needed to provide an on-site resource. Users only acquire a third-party license or access and start working simply like installation of the hardware locally.

\section{Disadvantage}

Once virtualization is considered, the cost for the ordinary individual or business is quite cheap. Leite et al. (2018) argued the implementing expenses might be very significant for suppliers of a virtualized environment. At some point, hardware and software are necessary and that implies that equipment must be designed, produced, or acquired for installation. While saving time during the virtualization phase, it costs users long-term time compared with native systems. On the other hand, there are more actions that have to be taken for the desired outcome to be generated in a proper manner.

\section{Utilisation of virtualisation in hardware and software}

As mentioned by Leite et al. (2018), Storage virtualization is the combination of physical storage from several network storage devices into one device that seems to be handled from a central interface. Moreover, Virtualization for storage is widespread in networks with storage areas as this process can support a computer system by prodigy many different kinds of strong and sustainable systems such as high levels of social security. In addition, virtualization on server's masks server resources, including the quantity and identity of individual servers, CPUs, and server operating systems. The purpose is to avoid complex server resource data being understood and managed while boosting the usage and use of resources and retaining the capacity to expand later.

In both enterprises operating private cloud computing and service providers, virtual machinery complicates IT security greatly. In the absence of security to secure a physical server or server collection on which an application operates, it can directly enhance the quality of the security protection in a proper manner. As per the words of Gryaznova et al. (2019), Virtualization defines technology that removes the actual underlying hardware or software from an app, host computer, or data storage. Server virtualization employs the software layer dubbed virtualization as it emulates the hardware devices as a major utility of server virtualization. On the other hand, the memory feedback and network traffic of the CPU typically are included and it also can be supportive for hypervisors use and separate physical resources to use the virtual environment.

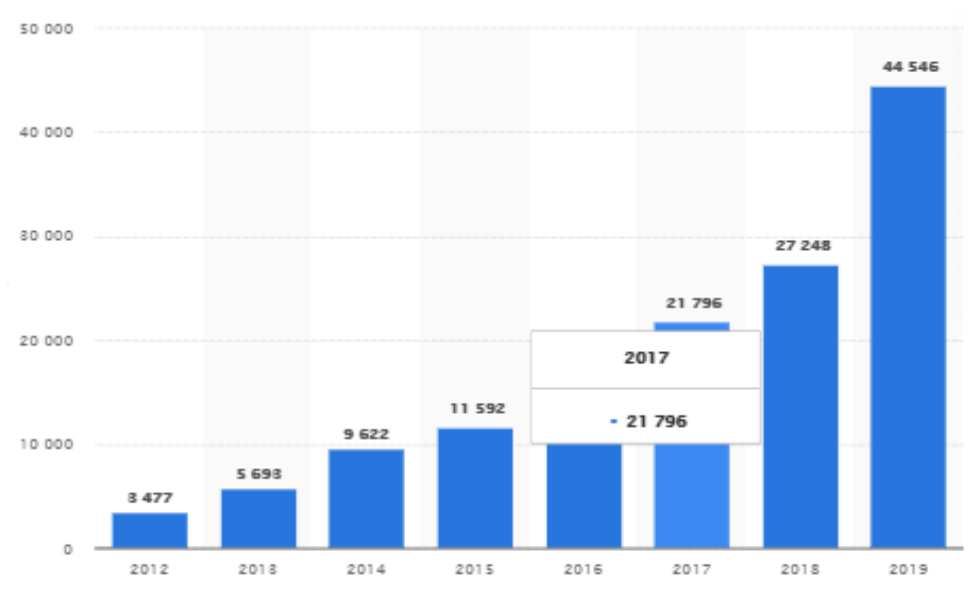

Figure 1: Cyber-crime rates in India since 2012-2019

(Source: Statista.com, 2019) 
Current protections for the network are based on physical networks as the network is no longer physically in a virtualized environment; in fact, its settings might change dynamically, making it impossible to monitor the network. Users must need to be responsible to observe virtual networks and, eventually, dynamic virtual networks in order to solve this challenge. As mentioned by Banushri and Karthika (2018), once the program is locked in virtual images, the simple act of changing its settings or patches of the software on virtual machines becomes considerably difficult in the virtual world, there are no longer static fixed addresses for updating its configuration. Sherwin and Sreenan, (2021) argued virtualization is a technical revolution, separating functions and underlying hardware and enabling us to build a useful and abstract environment. However, the technology of virtualization was attacked by hostile attackers and VM infrastructures might be compromised by attackers, such as they can access other VMs with the same system and host.

\title{
Effect of virtualization in running a virtual instance of the computer system from the actual hardware
}

The idea of virtualization has expanded to programs, networks, data, and desktops through many different kinds of virtual servers. While its efficiency is not the same as the android because android systems are running on real hardware, and the virtualization idea works underlying hardware completely. As per the suggestions of Bernstein (2019), these processes simply enable flexibility, monitoring, and insulation through the removal of dependence on a particular hardware platform. However, there are many different kinds of strong and sustainable steps are presented such as Physical sources are separated from the physical environment by hypervisors. Resources are collected from the actual environment and split, as necessary, into several virtual worlds. In the virtual world, system users operate and execute computations and the virtual environment is functioning, and a user or application can send the physical environment a message requiring additional resources. In addition, the hypervisor, in response, transmits the communication and saves the modifications to the physical system as it will occur at nearly indigenous speed.

\section{Methodology}

\section{Descriptive style}

\section{- Representation of data in a logical manner}

\section{Realism philosophy}

- Gain knowledge of current cyber crime rates in India

\section{Secondary qualitative reserach methods}

- Secondary data from jouranls, news articles and others

\author{
Figure 2: Methodology
}

(Source: Martins 2020)

In order to conduct any kind of research study methods and techniques proper presentation is one of the most important things. As per the suggestions of Martins (2020), the research methodology shall identify, select, process, and analyse material on the issue via the precise processes or techniques utilized. The aim of a research design is to produce a study plan that enables an accurate evaluation of connections between causes and effects between independent and dependent variables. This paper simple done through descriptive style as it supports the researcher to represent valid data in a logical manner. In addition, the selection of appropriate facts and objective methodologies for judging ideas and suggestions is highly emphasised in philosophical thought. This research paper simply adopts realism research philosophy as it supports the researcher to gain proper understandings about current situation of India's cybercrime rate. 
On the other hand, a research paper enables the reader to assess the overall validity and dependability of a study critically. This research paper will be done by a secondary qualitative research method as it supports the researcher to represent many different types of strong and sustainable viewpoints about the virtualization process that supports a computer system in a proper manner.

\section{Data Analysis \& Findings}

\section{Impacts of Virtualization}

Virtualization can bring concrete advantages, such as making it ultimately feasible to accomplish more with less. As an IT organization, it is an open conduction plot that simply embraces server virtualization technologies. Liu et al. (2021), explain why virtualization of servers has three distinct problems: breakdown of IT services; probable increases in security risks, and more complexity in the administration of change. On the other hand, modern virtualization technologies allow effective utilization of underutilized hardware and software by distributing them on the same virtual computer amongst virtual computers. These technologies have therefore been widely used in various fields, such as cellular connectivity, virtualized playable demo beds, and so on. One of the important instruments for the evaluation or verification of modern communication technology is a global network testbed.

However, the tested should fulfill the requirements of insulation, fidelity, repeatability, scalability, confinement, and extensibility if a network testbed is constructed which matches as much as a genuine configuration. The contemporary virtualization technology can achieve scalability among these criteria, however, the vulnerability and safety weakness, together with virtualization, may also be damaging to other requirements of a network testbed, such as insulation and loyalty.

\section{Importance of IoT in virtualization process}

The adoption of the I-IoT domain virtualization involves a number of possible advantages. However, the use of the virtualization platforms must be verified in the context of I-IoT if virtualization, which is now an established technology, is to be achieved. In this paper, we offer a quantitative comparison of two major open-source hypervisors, XEN and KVM, concentrating on the I-IoT output and latency. As mentioned by Gürdür et al. (2019), the IT sector has seen the most growth in recent times in the Internet of things and cloud computing, and this trend should continue. We are also witnessing nowadays an expanding number of different firms more interested in adopting IoT technology.

This tendency leads to a new smart Grid model that targets a number of existing commercial tools and procedures such as production, logistics, diagnostics, and adhesive bonding. Many organizations choose to save expenses and improve their servers' effectiveness with the use of private cloud computing in order to deliver I-IoT services in a cost-efficient manner. However, strengthening the subsystems into one server can reduce much complexity from the final system, simplify management and replacements. Virtualization also benefits from the fact that developers do not need to rewrite application code that matches the new hardware.

\section{Conclusion}

In order to represent the conclusion, it can be stated that virtualization uses hardware capability replicating software as this allows IT companies to run various operating systems and applications on a single server and several virtual systems. Moreover, the advantages of virtualization also include more efficiency and efficiency. In addition, OS Virtualization uses software to allow a device to run many OS images concurrently and the approach began decades earlier and allowed management to avoid the loss of expensive computing capacity. However, virtualization also decreases the quantity of the hardware servers necessary inside the organization and data center, reducing the total purchase cost and preserving enormous amounts. 


\section{References}

1. Bernstein, D.J., 2019. Visualizing size-security tradeoffs for lattice-based encryption. IACR Cryptol. ePrint Arch., 2019 , p.655.

2. Chatzimparmpas, A., Martins, R.M., Jusufi, I. and Kerren, A., 2020. A survey of surveys on the use of visualization for interpreting machine learning models. Information Visualization, 19(3), pp.207-233.

3. Gryaznova, E.V., Vladimirov, A.A., Maltceva, S.M., Goncharuk, A.G. and Zanozin, N.V., 2019, May. Problems of virtualization and internalization of social space. In Institute of Scientific Communications Conference (pp. 119-124). Springer, Cham.

4. Gürdür, D., El-khoury, J. and Nyberg, M., 2019. Methodology for linked enterprise data quality assessment through information visualizations. Journal of Industrial Information Integration, 15, pp.191-200.

5. Leite, R.A., Gschwandtner, T., Miksch, S., Gstrein, E. and Kuntner, J., 2018. Visual analytics for event detection: Focusing on fraud. Visual informatics, 2(4), pp.198-212.

6. Liu, S., Liu, L., Yang, N., Mao, D., Zhang, L., Cheng, J., Xue, T., Liu, L., Yan, G., Qiu, L. and Chen, X., 2021. A data-driven approach for online dynamic security assessment with spatial-temporal dynamic visualization using random bits' forest. International Journal of Electrical Power \& Energy Systems, 124, p.106316.

7. Manjushri, A. and Karthika, R.A., 2018. Implementation levels of virtualization and security issues in cloud computing. International Journal of Engineering \& Technology, 7(2.33), pp.678-682.

8. Muchagata, J. and Ferreira, A., 2018, March. How can visualization affect security? In ICEIS (2) (pp. 503-510).

9. Sherwin, J. and Sreenan, C.J., 2021. Software-Defined Networking for Data Centre Network Management: A Survey. arXiv preprint arXiv:2106.10014.

10. statista.com, 2019. The number of cybercrimes reported across India from 2012 to 2019. Available at: [Online] https://www.statista.com/statistics/309435/india-cyber-crime-it-act/ 